\title{
Modified Technique for Internal Ureteroileal Stenting in Orthotopic Neobladders
}

\author{
Guven Aslan \\ Dokuz Eylul University, School of Medicine Department of Urology, Izmir, Turkey \\ E-mail:aslang@deu.edu.tr \\ Received February 9, 2011; revised February 28, 2011; accepted March 31, 2011
}

\begin{abstract}
We introduce a modified surgical technique for internal ureteroileal stenting to improve cosmesis in patients undergoing ileal neobladder. Internal ureteric stents are secured to the tip of the urethral catheter with nonabsorbable suture facilitating removal of the stents postoperatively 2 - 3 weeks along with the urethral catheter. This surgical modification was applied to 21 patients of whom no significant surgical or infectious complication due to single urethral catheter was observed. Internal ureteral stenting is simple, safe and inexpensive alternative to conventional methods to support ureteroileal anastomosis in patients undergoing orthotopic bladder substitution.
\end{abstract}

Keywords: Bladder Cancer, Radical Cystectomy, Bladder Substitution, Ureteric Stent

\section{Introduction}

Radical cystectomy and urinary diversion represents the standard treatment for muscle invasive and non muscle invasive bladder cancer not controlled by conventional treatment options. The use of the orthotopic bladder substitution has gained general acceptance within the past decade and different types of surgical techniques have been described in this regard. An orthotopic ileal neobladder replacement is commonly used in both men and women [1]. The tendency for orthotopic neobladder is due to an increasing importance of providing patients better quality of life and a favorable cosmetic effect [2].

Because cystectomy is a major surgery with complex surgical procedures; the patients carry several bags for drains, external catheters and cystostomy tube which may cause restriction of patient postoperatively. Minor surgical modifications have been reported including omitting gastrostomy or cystostomy tube, and internalizing the ureteral stents as the experiences with any diversion technique increased over the decades since they have been first popularized [2-4]. We present our surgical experience with modification of internal stenting for bladder substitution.

\section{Technique}

The indications and surgical techniques for open radical cystectomy, including extended pelvic lymph node dis- section, have been described previously [5]. Diversion types performed were either T Pouch or Studer type urinary diversion as described previously [6,7]. The orthotopic bladder substitute was performed by isolating 50 - $60 \mathrm{~cm}$ of ileum, $25 \mathrm{~cm}$ proximal to the ileocecal valve. Both ureters were isolated. The left ureter was mobilised up to the lower pole of the kidney with care to maintain its surrounding blood supply. The left ureter was transposed to the right side through a retrosigmoidal tunnel.

Once the pouch has been closed, each ureter was spatulated and a standard, bilateral end-to-side ureteroileal anastomosis was made to the proximal portion of the afferent limb using interrupted 4 - 0 polyglycolic acid suture. Prior to completion of the anastomoses each ureter was stented with a $8 \mathrm{~F}$ feeding catheter (8F Levin's Tube $65-75 \mathrm{~cm}$ long) that is cut open-ended both side. These anastomoses were stented with $8 \mathrm{~F}$ infant-feeding tubes, which are directed from the ipsilateral renal pelvis, across the uretero-ileal anastomosis through the afferent limb into the reservoir and out of the pouch (Figures 1 (a) - (d)). A 22 - $24 \mathrm{~F}$ foley catheter was placed urethrally to provide adequate drainage of the reservoir and the ureteric stents were secured to the tip of the urethral catheter with nonabsorbable 0 Prolene sutures (Figures 2 (a) (d)). This facilities removal of the stents at postoperatively 3 weeks, along with the urethral catheter. A tension free mucosa-to-mucosa urethro-ileal anastomosis was made at the end of procedure. 

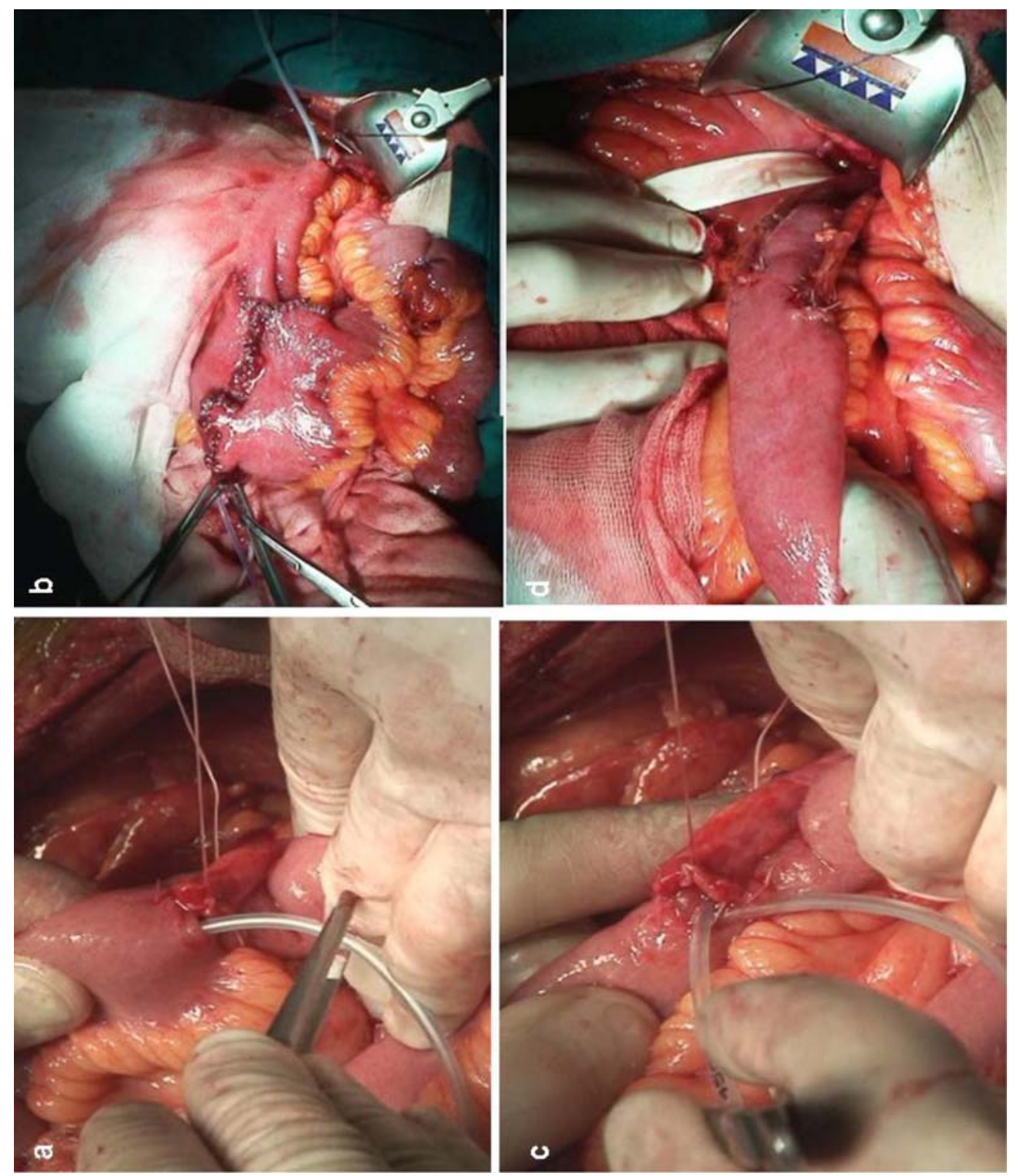

Figure 1. (a) ureteral stent introduced to afferent limb of reservoir where ureter anostomosis is done; (b) ureteral stent passed through the reservoir to the distal open end of neobladder and grasped by atraumatic Allis clamp; (c) Proximal 20 $25 \mathrm{~cm}$ of catheter segment directed to the kidney through ureter; (d) both ureteric end-to-side anastomosis are completed.

We routinely do not use an additional suprapubic cystostomy tube. Our irrigation protocol consisted of daily irrigations every $4 \mathrm{~h}$ in order to keep the neobladder free from accumulated mucus. The frequency of irrigations was tapered down during the first postoperative week. All patients were trained by the nursing staff how to irrigate their neobladder twice daily until the catheter was removed. Patients were followed up at 2 weeks and 1, 3 months postoperatively and continued every 6 months thereafter.

\section{Results}

From 2006 to 2010, 21 (19 males and 2 females) with a median age of 59 (54 - 66) years underwent orthotopic ileal neobladder operation. The median total operating time was 330 (270 - 390) minutes. The median blood loss was $500 \mathrm{~mL}$ (400 - $1000 \mathrm{~mL})$. The median number of lymph nodes removed was 19 (11 - 29). No intraoperative adverse event was observed in any patient.

Prolonged urinary leak from the ureteroilial anastomosis (15 days postoperatively) was observed in one patient which ceased after insertion of percutaneous nephrostomy catheter and recovered with no further intervention. No cystogram was performed before removing the Foley catheter. The median hospital stay was 11 (7 - 21) days. Postoperative ileus developed in 5 (23.8\%) patients. No patient needed surgical intervention for ileus and all 

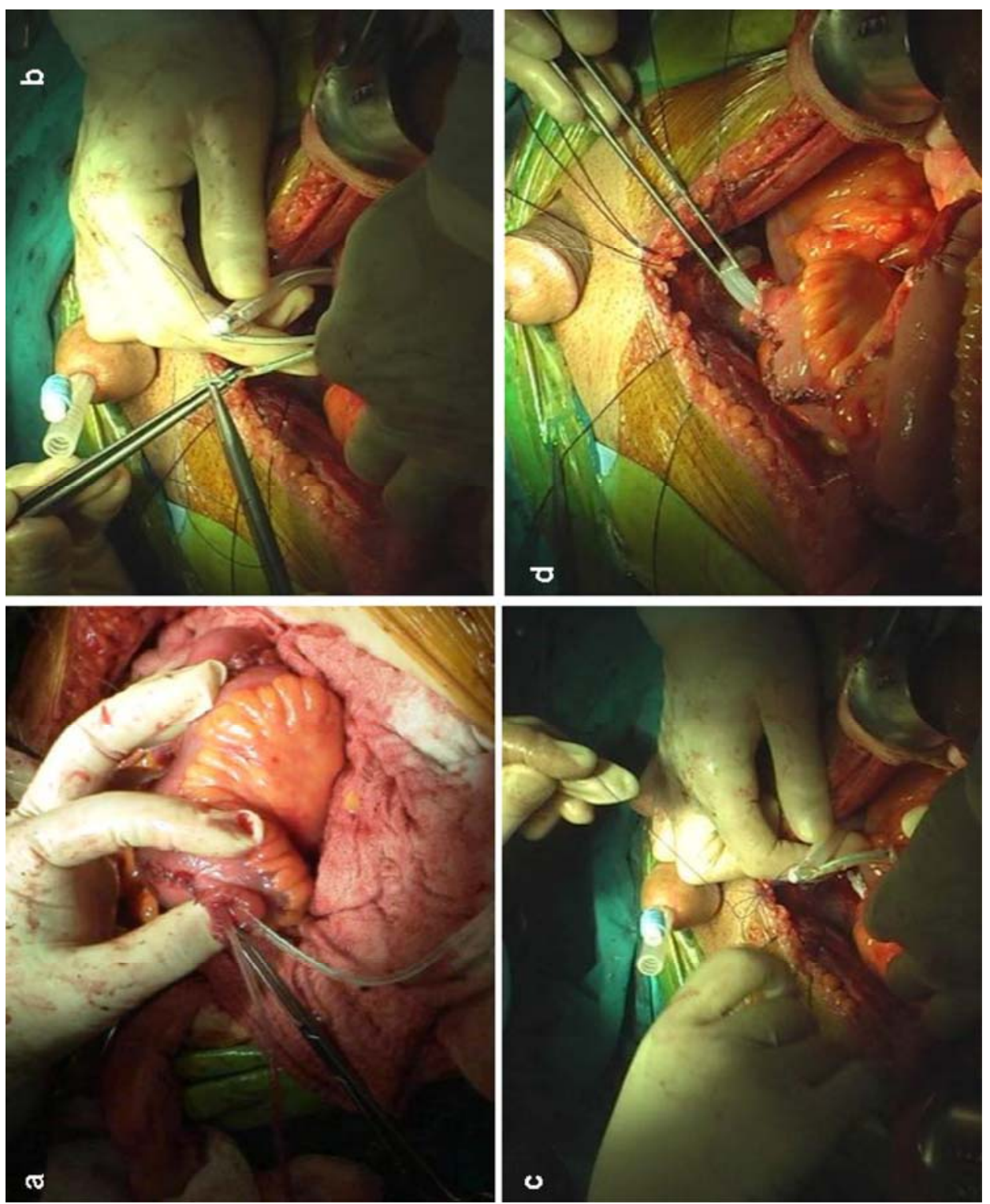

Figure 2. (a) both ureteric stents passed through the afferent limb into the reservoir and out of the neourethra; (b) Stents are secured to the tip of the catheter with non absorbable suture; (c) tied; (d) Foley catheter and anchored stents directed to the reservoir to create single urethral catheter drainage.

episodes of ileus resolved with standard treatments [8]. There was no bowel anastomosis leakage. No documented urinary tract infection determined in any patient. Stent migration, premature dislodgement, stent occlusion or incrustation was not observed in any patient. We have not determined any Foley catheter blockage due to plug and Foley catheters were not changed in any patient. Ureteral stents could be taken out easily by pulling out the Foley catheter at 2 - 3 weeks.

Median follow-up time was 3 years ( 2 - 4 years). Strictures of the ureteroileal anastomosis occurred in 2 of patients in the first postoperative year and were treated with open reimplantation.

\section{Discussion}

Stenting of the ureteroileal anastomosis has been shown to have significant beneficial effect with decreased early postoperative upper urinary tract dilatation, improved recovery of bowel function and decreased metabolic acidosis [9]. Conventionally both open-ended straight ureteral catheters, brought out through a stab wound in the reservoir and anchored to the skin are typically used for ureteroileal stenting in orthotopic neobladders $[2,6]$. In addition to ureteric splints cystostomy tubes for neobladder, urethral catheter, drains and gastrostomy tube (some of centers) taken out externally through the 
abdominal wall and therefore, the patients have to carry several bags, with resultant of patient restriction and discomfort. Apart from cosmesis and patient acceptability suprapubic tubes may cause morbidity such as stent migration, urinary leak when they were taken out from the neobladder or even persistent neobladder fistula [10]. Thus several modifications of techniques have been proposed those for tubes and ureteric stents [3,4]. Internal ureteral stents anchored to foley catheter as we show here is described elsewhere; however no published long term results of this technique exist since then [11]. More recently double $\mathrm{J}$ stents have been favored as internal splint across the ureteroilial anastomosis with good results $[3,4]$. However, the disadvantages of this internal stenting are the need to remove the stents with cystoscope and their expensiveness compared to feeding tubes. More recently similar to the technique we used double $\mathrm{j}$ stents are anchored to the Foley catheter as urinary drainage with single urethral catheter to be removed at 3 weeks [4].

The technique we used herein did not lead to any untoward events. We have not observed any significant surgical or infectious complication or any catheter related problem due to single urethral catheter. There is certain risk of catheters getting blocked. However with frequent foley irrigation postoperatively this may be avoided. We used infant feeding catheter as ureteral stent with open ended both sides. When anyway foley catheter or ureter catheter need to be changed postoperatively; Foley can be pulled slowly to level 1 - $2 \mathrm{~cm}$ distal to external meatus then a guidewire can be inserted through the open end of ureteral stent and a catheter can be inserted over the guidewire fluoroscopically.

Internal ureteral stenting is simple, safe and inexpensive alternative to conventional methods for supporting of the ureteroileal anastomosis in patient undergoing orthotopic bladder substitution.

\section{References}

[1] R. E. Hautmann, B. G. Volkmer, M. C. Schumacher, J. E. Gschwend and U. E. Studer, "Long-Term Results of Standard Procedures in Urology: The Ileal Neobladder,”
World Journal of Urology, Vol. 24, No. 3, 2006, pp. 305-314. doi:10.1007/s00345-006-0105-Z

[2] R. Thurairaja, F. C. Burkhard and U. E. Studer, "The Orthotopic Neobladder,” British Journal of Urology International, Vol. 102, 2008, pp. 1307-1313.

[3] I. M. Varkarakis, A. Delis, A. Papatsoris and C. Deliveliotis, "Use of External Ureteral Catheters and Internal Double J Stents in a Modified Ileal Neobladder for Continent Diversion: A Comparative Analysis,” Urologia Internationalis, Vol. 75, No. 2, 2005, pp. 139-143. doi:10.1159/000087168

[4] A. Mandhani, A. Dharaskar and R. Kapoor, "Technical Steps of Open Radical Cystectomy and Orthotopic Neobladder to Achieve the Goals of 'Minimally Invasive Surgery'?” Indian Journal of Urology, Vol. 26, No. 3, 2010, pp. 457-460. doi:10.4103/0970-1591.70596

[5] J. P. Stein and D. G. Skinner, "Radical Cystectomy," British Journal of Urology International, Vol. 94, 2004, pp. 197-221.

[6] U. E. Studer, C. Varol and H. Danuser, "Orthotopic Ileal Neobladder,” British Journal of Urology International, Vol. 93, 2004, pp. 183-193.

[7] J. P. Stein and D. G. Skinner, "The Orthotopic T-Pouch Ileal Neobladder," British Journal of Urology International, Vol. 98, 2006, pp. 469-482.

[8] B. Pearson and S. D. Wexner, "The Management of Postoperative Ileus,” Current Problems in Surgery, Vol. 43, No. 1, 2006, pp. 12-65.

[9] A. Mattei, F. D. Birkhaeuser, C. Baermann, S. H. Warncke and U. E. Studer, "To Stent or Not to Stent Perioperatively the Ureteroileal Anastomosis of Ileal Orthotopic Bladder Substitutes and Ileal Conduits? Results of a Prospective Randomized Trial,” Journal of Urology, Vol. 179, No. 2, 2008, pp. 582-586. doi:10.1016/j.juro.2007.09.066

[10] H. M. Abd Alla and A. A. Attia, "Lower Urinary Tract Reconstruction Following Radical Cystectomy Using Ileal Neobladder with Studer Technique; 3 Years Experience,” Journal of the Egyptian National Cancer Institute, Vol. 12, No. 4, 2000, pp. 235-243.

[11] S. D. Mark and G. D. Webster, "Simplified Urinary Drainage Following Orthotopic or Continent Bladder Replacement,” Journal of Urology, Vol. 153, No. 2, 1995, pp. 334-335. doi:10.1097/00005392-199502000-00010 